PROCEEDINGS OF THE AMERICAN MATHEMATICAL SOCIETY

Volume 124, Number 2, February 1996

\title{
A NOTE ON THE WEIGHTED NORM INEQUALITY FOR THE ONE-SIDED MAXIMAL OPERATOR
}

\author{
LAI QINSHENG
}

(Communicated by J. Marshall Ash)

\begin{abstract}
Let $M_{g}^{+}$be the one-sided maximal function. In this note we obtain some necessary and sufficient conditions in order that the weighted weak type inequality holds for $M_{g}^{+}$. Meanwhile, some necessary or sufficient conditions for the weighted inequality for $M_{g}^{+}$are given.
\end{abstract}

\section{INTRODUCTION}

Given a positive and locally integrable function $g$ on the real line $R$, the one-sided maximal function $M_{g}^{+}$is defined by

$$
M_{g}^{+} f(x)=\sup _{h>0} \frac{1}{g(x, x+h)} \int_{x}^{x+h}|f(y)| g(y) d y
$$

for $f \in L_{\text {loc }}^{1}(g(x) d x)$, where $g(x, x+h)=\int_{x}^{x+h} g(y) d y$.

Symmetrically, the left maximal function $M_{g}^{-}$can be defined in the obvious way.

Recently, weighted inequalities for the operators $M_{g}^{+}$and $M_{g}^{-}$have been extensively studied (see [9], [6], [5], [4]). In this note we will characterize the pairs of weights $(w, v)$ such that $M_{g}^{+}$(or $\left.M_{g}^{-}\right)$is of weak type $(1, q)$ with $1 \leq q$. Secondly, we will give a necessary condition on the pairs $(w, v)$ in order that the weak type $(p, q)$ inequality with $0<p \leq q<\infty$ holds for $M_{g}^{+}$(or $M_{g}^{-}$) with respect to the measures $w d x$ and $v d x$. This result shows that most weight functions do not verify the weighted weak type $(p, q)$ inequality when $1<p<q<\infty$. In the case of $1 \leq p$ and $0<q<p$ we will introduce a $B_{g}^{+}(p, q)$ ( or $B_{g}^{-}(p, q)$ ) condition and prove that this condition is sufficient for the weak type $(p, q)$ inequality and necessary for the strong type $(p, q)$ inequality for the operator $M_{g}^{+}$(or $M_{g}^{-}$). Finally, we will prove analogies of Sawyer's theorem in [10] and Verbitsky's theorem in [11]. They characterize the weak type $(p, q)$ inequality when $1 \leq p<\infty$ and $0<q<p$.

Throughout this paper, $w$ and $v$ always are weight functions by which we mean nonnegative measurable functions taking values in $[0, \infty]$. For a given weight $w$ and measurable set $E, w(E)=\int_{E} w(x) d x$ and $\chi_{E}(x)$ denotes the characteristic function of $E$. Particularly, $|E|$ is the Lebesgue measure of $E$ and $w((a, b))$ is written by $w(a, b)$. For $p \geq 1$, let $p^{\prime}=\frac{p}{p-1}$ be its conjugate index, and we adopt the usual

Received by the editors January 28, 1993 and, in revised form, September 7, 1994.

1991 Mathematics Subject Classification. Primary 42B25.

Key words and phrases. One-sided maximal function, weighted inequality, weight.

(C)1996 American Mathematical Society 
conventions for multiplication in $[0, \infty]$, namely, $\infty \cdot t=t \cdot \infty=\infty$ for $0<t \leq \infty$, $0 \cdot \infty=\infty \cdot 0=0, \infty^{-1}=0$ and $0^{-1}=\infty$.

Now we state our results. We just present our theorems concerning $M_{g}^{+}$, since their counterparts for $M_{g}^{-}$can be obtained similarly.

Theorem 1. Suppose $1 \leq q<\infty$. Then the weak type $(1, q)$ inequality

$$
\left(\lambda^{q} w\left(\left\{x \in R: M_{g}^{+} f(x)>\lambda\right\}\right)\right)^{1 / q} \leq C \int_{R}|f(x)| v(x) d x
$$

holds for all $\lambda>0$ and measurable $f$, if and only if $(w, v) \in A_{g}^{+}(1, q)$, that is,

$$
\sup _{a<b<c}\left(\frac{w(a, b)^{1 / q}}{g(a, c)} \operatorname{essup}_{x \in(b, c)}\left(\frac{g}{v}\right)\right)=A<\infty .
$$

Moreover, for the best constants $A$ and $C$, we have $A \leq C \leq 4 A$.

Remark 1 . The good weights for the weak type $(p, p)$ inequalities $(p \geq 1)$ were established in [6], [9] and [5]. However, in those articles the condition $A_{g}^{+}(1,1)$ was represented by

$$
M_{g}^{-}\left(\frac{w}{g}\right)(x) \leq C \frac{v(x)}{g(x)} \quad \text { for a.e. } x \in R .
$$

Later on, in [4], we established the weak type inequalities for $M_{g}^{+}$in Orlicz classes. In the particular case of $1<p \leq q<\infty$ we obtained the $A_{g}^{+}(p, q)$ condition, i.e.

$$
\sup _{a<b<c} \frac{w(a, b)^{1 / q} \sigma(b, c)^{1 / p^{\prime}}}{g(a, c)}=A<\infty,
$$

where $\sigma=g^{p^{\prime} 1} v^{1-p^{\prime}}$. Our $A_{g}^{+}(1, q)$ condition can be considered as the limiting case of $A_{g}^{+}(p, q)$ as $p \rightarrow 1$. Furthermore, it has an extended version in the case $0<q<p=1$ (cf. Theorem 4). This sort of $A_{g}^{+}(1, q)$ conditions for $M_{g}^{+}$are new in the literature, although the parallel conditions for usual Hardy-Littlewood maximal operator are well known.

Theorem 2. Let $0<p \leq q<\infty$ and $(w, v)$ is a pair of weight functions. Suppose the weak type $(p, q)$ inequality

$$
\left(\lambda^{q} w\left(\left\{x \in R: M_{g}^{+} f(x)>\lambda\right\}\right)\right)^{1 / q} \leq C\left(\int_{R}|f(x)|^{p} v(x) d x\right)^{1 / p}
$$

holds for all $\lambda>0$ and measurable $f$. Then we have:

(i) For a.e. $x \in R$, either $w(x)=0$ or $v(x)=\infty$ if $p<q$.

(ii) There exists a constant $B$ such that

$$
w(x) \leq B v(x) \quad \text { a.e. } x \in R
$$

if $p=q$. Moreover, $B \leq(2 C)^{p}$ for the best constants $B$ and $C$ in (1.6) and (1.5) respectively.

Remark 2. Theorem 2 indicates that if $v$ is finite a.e. (e.g. locally integrable) and $p<q$, then the weight $w$ must be zero a.e. But, the $A_{g}^{+}(p, q)(1 \leq p \leq q<\infty)$ always contain nontrivial pairs of weight functions, i.e. neither $w(x) \equiv 0$ nor $v(x) \equiv$ $\infty$. Here we shall give an example. 
Example 1. Since $g$ is locally integrable, there exists $M>0$ such that

$$
|E|=|\{x \in R: g(x) \leq M\}|>0 .
$$

Choose an interval $\left(a_{1}, a_{2}\right)$ satisfying $\left|\left(a_{1}, a_{2}\right) \cap E\right|>0$, and fix $a_{3}<a_{4}<a_{1}$ arbitrary. Let $w(x)=\chi_{\left[a_{3}, a_{4}\right]}(x), v(x)=1$ for $x \in\left(a_{1}, a_{2}\right) \cap E$ and $v(x)=\infty$ elsewhere. Then $(w, v) \in A_{g}^{+}(p, q)$ for all $1 \leq p \leq q<\infty$.

Indeed, we only need to verify the $A_{g}^{+}(p, q)$ conditions for $a<b<c$ with $(a, b) \cap$ $\left(a_{3}, a_{4}\right) \neq \varnothing$ and $(b, c) \cap\left(a_{1}, a_{2}\right) \neq \varnothing$. In these cases, it is obvious that $g(b, c) \geq$ $g\left(a_{4}, a_{1}\right), w(a, b) \leq a_{4}-a_{3}, \sigma(b, c) \leq M^{p^{\prime}}\left(a_{2}-a_{1}\right)(p>1)$ and $\operatorname{ess} \sup _{x \in(b, c)} \frac{g(x)}{v(x)} \leq$ $M(p=1)$. Then the conclusion follows easily.

Definition 1. Suppose $1 \leq p<\infty$ and $0<q<p$. We say that a pair of weight functions $(w, v) \in B_{g}^{+}(p, q)$ if

$$
\begin{gathered}
{\left[\sum_{j}\left(\frac{w\left(a_{j}, b_{j}\right)^{1 / q} \sigma\left(b_{j}, c_{j}\right)^{1 / p^{\prime}}}{g\left(a_{j}, c_{j}\right)}\right)^{r}\right]^{1 / r} \leq B<\infty \quad(\text { when } p>1),} \\
{\left[\sum_{j}\left(\frac{w\left(a_{j}, b_{j}\right)^{1 / q}}{g\left(a_{j}, c_{j}\right)} \operatorname{ess~sup}_{x \in\left(b_{j}, c_{j}\right)}\left(\frac{g(x)}{v(x)}\right)\right)^{r}\right]^{1 / r} \leq B<\infty \quad(\text { when } p=1)}
\end{gathered}
$$

for all sequences $\left\{\left(a_{j}, c_{j}\right)\right\}$ of pairwise disjoint intervals and $b_{j} \in\left(a_{j}, c_{j}\right)$. Where $\sigma=\sigma_{g, v, p}=g^{p^{\prime}} v^{1-p^{\prime}}$ and $\frac{1}{r}=\frac{1}{q}-\frac{1}{p}$. We shall keep these notations throughout this paper.

Theorem 3. Suppose $1 \leq p<\infty, 0<q<p$ and $(w, v)$ is a pair of weight functions. If $(w, v) \in B_{g}^{+}(p, q)$, then the weak type $(p, q)$ inequality (1.5) holds for all $\lambda>0$ and measurable $f$. Moreover, for the best constants $B$ in Definition 1 and $C$ in (1.5) we have $C \leq 2^{2+1 / p^{\prime}} B$.

On the other hand, if the strong type $(p, q)$ inequality

$$
\left(\int_{R}\left[M_{g}^{+} f(x)\right]^{q} w(x) d x\right)^{1 / q} \leq C\left(\int_{R}|f(x)|^{p} v(x) d x\right)^{1 / p}
$$

holds for all measurable $f$, then $(w, v) \in B_{g}^{+}(p, q)$ with $B \leq C$.

Remark 3. We will give some examples of $B_{g}^{+}(p, q)$ functions, which shows that the $B_{g}^{+}(p, q)$ condition is not sufficient for the strong type inequality.

Example 2. Suppose $(u, v) \in A_{g}^{+}(p, p)(p \geq 1)$. Choose $\Omega \in \mathbf{R}$ with $u(\Omega)<\infty$. Let $w=u \chi_{\Omega}$. Then $(w, v) \in B_{g}^{+}(p, q)$ for all $0<q<p$.

Example 3. Choose $\Omega \subset \mathbf{R}$ bounded. Let $w=g$, and $v=g$ on $\Omega$ and $\infty$ elsewhere. Then $(w, v) \in B_{g}^{+}(p, q)$ for all $1 \leq q<p$. Particularly, setting $g=1$ and $\Omega=[0,1]$, we obtain a simple example which satisfies $B_{g}^{+}(p, 1)$ (therefore the weak type $(p, 1)$ inequality), but the function $f=\chi_{\Omega}$ causes the strong type $(p, 1)$ inequality to fail.

For $q>1$, an example follows from the corresponding example given by Muckenhoupt in the case $p=q=2$ (see [7], p. 218).

Example 4. Set $g=1$. Let $w(x)=x \log (-x)$ on $\left[-\frac{1}{2}, 0\right)$ and 0 elsewhere, and $v(x)=x^{2}(\log (-x))^{4}$ on $\left[-\frac{1}{2}, 0\right)$ and $\infty$ elsewhere. Then $(w, v) \in B_{g}^{+}(3,2)$, but the function $f(x)=-\chi_{\left[-\frac{1}{2}, 0\right)}(x) /\left(x \log ^{2}(-x)\right)$ violates the strong type $(3,2)$ inequality. 
Definition 2. Suppose $1 \leq p<\infty$ and $0<q<p$. For every $(a, b) \subset \mathbf{R}$, let

$$
\begin{aligned}
& \rho_{(a, b)}=\sup _{c>b} \frac{w(a, b)^{1 / p} \sigma(b, c)^{1 / p^{\prime}}}{g(a, c)} \quad(p>1), \\
& \rho_{(a, b)}=\sup _{c>b} \frac{w(a, b)}{g(a, c)} \operatorname{ess~sup}_{x \in(b, c)}\left(\frac{g(x)}{v(x)}\right) \quad(p=1) .
\end{aligned}
$$

Define $\Phi(x)=\Phi_{p, q, w, v}(x)=\sup \left\{\rho_{(a, b)} \chi_{(a, b)}(x)\right\}$.

Theorem 4. Suppose $1 \leq p<\infty$ and $0<q<p$. The following statements are equivalent.

(i) The weak type inequality (1.5) holds.

(ii) There exists a constant $B>0$ such that for every sequence $\left\{\left(a_{j}, b_{j}\right)\right\}$ of pairwise disjoint $\left(a_{j}, b_{j}\right)$ and $c_{j}>b_{j}$ arbitrary, the inequality

$$
w\left(\bigcup_{j}\left(a_{j}, b_{j}\right)\right)^{1 / q} \leq B\left\{\sum_{j}\left(\frac{g\left(a_{j}, c_{j}\right)}{\sigma\left(b_{j}, c_{j}\right)^{1 / p^{\prime}}}\right)^{p}\right\}^{1 / p} \quad(p>1)
$$

or

$$
w\left(\bigcup_{j}\left(a_{j}, b_{j}\right)\right)^{1 / q} \leq B \sum_{j} \frac{g\left(a_{j}, c_{j}\right)}{{\operatorname{ess} \sup _{x \in\left(b_{j}, c_{j}\right)}\left(\frac{g(x)}{v(x)}\right)}^{1 / q}} \quad(p=1)
$$

holds.

(iii) The function $\Phi(x)$ defined in Definition 2 is in $L^{r, \infty}(w)$, i.e.

$$
\|\Phi\|_{r, \infty, w}=\sup _{\lambda>0} \lambda w(\{|\Phi(x)|>\lambda\})^{1 / r}<\infty .
$$

Moreover, for the best constants $B$ and $C$ we have

$$
2^{-1 / r}\|\Phi\|_{r, \infty, W} \leq B \leq C \leq\left(\|\Phi\|_{r, \infty, W}^{r}+4^{p}\right)^{1 / r} .
$$

In spite of Theorem 4 , Theorem 3 is still worthwhile, because the $B_{g}^{+}(p, q)$ condition is more convenient in applications. For instance, it is not easy to test Examples 3 and 4 by condition (ii) or (iii).

Remark 4 . The equivalence between (i) and (ii) holds even for arbitrary $0<q<\infty$, and this is a Sawyer's type characterization for the one-sided maximal function (cf. [10]). The condition (iii) is a Verbitsky's type condition (cf. [11]). However, the case of $p=1$ is new and the proof is new.

\section{Proofs of the theOREMS}

Proof of Theorem 1. Necessity. The argument is based on the following elementary fact about the weak type $(p, q)$ inequality $(1.5)$.

For every $a<b<c$ and measurable set $S \subset(b, c)$, it follows from the weak type inequality (1.5) easily that

$$
\frac{g(S)}{g(a, c)} w(a, b)^{1 / q} \leq C v(S)^{1 / p},
$$

on setting $f(x)=\chi_{S}(x)$. 
Given $-\infty<a<b<c<\infty$, without loss of generality, we may assume $0<\operatorname{ess} \sup _{x \in(b, c)}\left(\frac{g}{v}\right)<\infty$. Then $0<\alpha_{g, v,(b, c)}=\alpha=\operatorname{essinf}_{x \in(b, c)}\left(\frac{v}{g}\right)<\infty$. Let $\lambda>\alpha$. It is obvious that

$$
\left|S_{\lambda}\right|=\left|\left\{x \in(b, c): \frac{v(x)}{g(x)}<\lambda\right\}\right|>0 .
$$

It follows from the inequality (2.1) that

$$
\frac{w(a, b)^{1 / q}}{g(a, c)} \leq C \frac{v\left(S_{\lambda}\right)}{g\left(S_{\lambda}\right)}<C \lambda
$$

Letting $\lambda \rightarrow \alpha$, we get

$$
\frac{w(a, b)^{1 / q}}{g(a, c)} \leq C \alpha .
$$

Sufficiency. We may assume that $f \geq 0$ is bounded and has compact support. Let $\lambda>0$ and $\Omega_{\lambda}=\left\{x: M_{g}^{+} f(x)>\lambda\right\}$. It is well known that (see [6]) $\Omega_{\lambda}=$ $\bigcup_{j}\left(a_{j}, b_{j}\right)$ where the intervals $\left(a_{j}, b_{j}\right)$ are bounded pairwise disjoint and

$$
\lambda \leq \frac{1}{g\left(x, b_{j}\right)} \int_{x}^{b_{j}} f(x) g(x) d x \quad \text { for every } x \in\left[a_{j}, b_{j}\right) .
$$

Following the idea used in [5], we set $x_{j}^{0}=a_{j}$ and $x_{j}^{k} \in\left(a_{j}, b_{j}\right)$ such that

$$
\int_{x_{j}^{k}}^{b_{j}} f(x) g(x) d x=\frac{1}{2^{k}} \int_{a_{j}}^{b_{j}} f(x) g(x) d x \quad(k=1,2, \ldots) .
$$

Then $\left\{x_{j}^{k}\right\}_{k}$ is increasing, $\lim _{k \rightarrow \infty} x_{j}^{k}=b_{j}$ and

$$
\lambda \leq \frac{4}{g\left(x_{j}^{k}, x_{j}^{k+2}\right)} \int_{x_{j}^{k+1}}^{x_{j}^{k+2}} f(x) g(x) d x .
$$

Then we have

$$
\begin{aligned}
\lambda^{q} w\left(\Omega_{\lambda}\right) & =\sum_{j} \sum_{k=0}^{\infty} \lambda^{q} w\left(x_{j}^{k}, x_{j}^{k+1}\right) \\
& \leq 4^{q} \sum_{j} \sum_{k=0}^{\infty} \frac{w\left(x_{j}^{k}, x_{j}^{k+1}\right)}{g\left(x_{j}^{k}, x_{j}^{k+2}\right)^{q}}\left(\int_{x_{j}^{k+1}}^{x_{j}^{k+2}} f g\right)^{q} \quad(\text { by }(2.4)) \\
& \leq 4^{q} \sum_{j} \sum_{k=0}^{\infty} \frac{w\left(x_{j}^{k}, x_{j}^{k+1}\right)}{g\left(x_{j}^{k}, x_{j}^{k+2}\right)^{q}}\left(\operatorname{ess~sup}_{x \in\left(x_{j}^{k+1}, x_{j}^{k+2}\right)}\left(\frac{g}{v}\right)\right)^{q}\left(\int_{x_{j}^{k+1}}^{x_{j}^{k+2}} f v\right)^{q} \\
& \leq(4 A)^{q} \sum_{j} \sum_{k=0}^{\infty}\left(\int_{x_{j}^{k+1}}^{x_{j}^{k+2}} f v\right)^{q}\left(\text { by } A_{g}^{+}(1, q) \text { condition }(1.3)\right) \\
& \leq\left(4 A \int f(x) v(x) d x\right)^{q} .
\end{aligned}
$$

This completes the proof of Theorem 1.

Remark 5 . The necessity of Theorem 1 is still true when $0<q<1$. An immediate consequence is this: if the weak type $(1, q)$ inequality holds for $M_{g}^{+}$with respect to $(w, v)$ and $\operatorname{ess}_{\sup }(a, b)\left(\frac{g}{v}\right)=\infty$, then $w(x)=0$ a.e. $x \in(-\infty, a)$. 
Proof of Theorem 2. The result is derived from the following propositions.

Proposition 1. Suppose weak type $(p, q)$ inequality (1.5) holds for given $0<p$, $q<\infty$. If $w$ is not locally integrable, then there exists $a \in[-\infty, \infty]$ such that $w$ is locally integrable on $(-\infty, a)$ and $v(x)=\infty$ a.e. $x \in(a, \infty)$. On the other hand, if $v(x)=0$ on $S$ with $|S|>0$, then there exists $b \in[-\infty, \infty]$ such that $w(x)=0$ a.e. $x \in(-\infty, b)$ and $v(x)>0$ a.e. $x \in(b, \infty)$.

Proposition 2. Under the same conditions as those in Theorem 2, suppose $v(x)>$ 0 a.e. and $w(x)$ is locally integrable on an interval $(a, b)$. Then we have

(i) For a.e. $x \in(a, b)$, either $w(x)=0$ or $v(x)=\infty$ if $p<q$.

(ii) $w(x) \leq(2 C)^{p} v(x)$ a.e. $x \in(a, b)$ if $p=q$. (Where $C$ is the best constant in (1.6).)

Proof of Proposition 1. Let

$$
E=\{x \in R: \text { there exists } \delta>0 \text { such that } w(x-\delta, x+\delta)<\infty\}
$$

and put $a=\inf \{R \backslash E\}$. It is obvious that $w$ is locally integrable on $(-\infty, a)$.

Meanwhile, for $a<c<d$ and measurable $S \subset(c, d)$ arbitrary, there exists $e \in R \backslash E$ with $a \leq e<c$. It follows from the definition of $R \backslash E$ that $w(e-\delta, c)=\infty$ for any $\delta>0$. Hence the inequality (2.1) shows that $v(S)=\infty$, which implies $v(x)=\infty$ a.e. $x \in(a, \infty)$, since $S \subset(c, d)$ and $(c, d) \subset(a, \infty)$ are arbitrary.

In order to prove the rest, we set $\widetilde{E}=\{x \in R: v(x)=0\}$. Let

$$
b=\operatorname{ess} \sup \left(I(x) \chi_{\widetilde{E}}(x)\right),
$$

where $I(x)=x$. For every $x<b$, we have $|\widetilde{E} \cap(x, b)|>0$. Then the inequality (2.1) implies $w\left(x_{1}, x\right)=0$ for all $x_{1}<x$. The required conclusion follows.

Proof of Proposition 2. In order to produce a contradiction to (i) we assume that there exists a constant $M>0$ such that

$$
|E|=\left|\left\{x \in(a, b): w(x)>\frac{1}{M}, v(x)<M\right\}\right|>0 .
$$

Write $v_{E}(x)=v(x) \chi_{E}(x)$, then $v_{E}$ is locally integrable. Choose $x_{0} \in E$ being a Lebesgue point of $g(x), w(x), v_{E}(x)$ and a point of density of $E$.

Since $x_{0}$ is a point of density of $E$, for every positive integer $n$, there exists a $h_{n}>0$ such that

$$
1-\frac{1}{n}<\frac{\left|E \cap\left(x_{0}-h_{n}, x_{0}+h_{n}\right)\right|}{2 h_{n}} \leq 1
$$

and $h_{n} \rightarrow 0$ as $n \rightarrow \infty$. For these $h_{n}$ we have

$$
\begin{aligned}
1-\frac{1}{n} & <\frac{\left|E \cap\left(x_{0}-h_{n}, x_{0}\right)\right|}{2 h_{n}}+\frac{\left|E \cap\left(x_{0}, x_{0}+h_{n}\right)\right|}{2 h_{n}} \\
& \leq \frac{1}{2}+\frac{\left|E \cap\left(x_{0}, x_{0}+h_{n}\right)\right|}{2 h_{n}},
\end{aligned}
$$

therefore

$$
\frac{1}{2}-\frac{1}{n}<\frac{\left|E \cap\left(x_{0}, x_{0}+h_{n}\right)\right|}{2 h_{n}} .
$$


Let $S=E \cap\left(x_{0}, x_{0}+h\right)$. It follows from (2.1) that

$$
\frac{g\left(E \cap\left(x_{0}, x_{0}+h_{n}\right)\right)}{g\left(x_{0}-h_{n}, x_{0}+h_{n}\right)} w\left(x_{0}-h_{n}, x_{0}\right)^{1 / q} \leq C v_{E}\left(x_{0}, x_{0}+h_{n}\right)^{1 / p} .
$$

By use of (2.5) we have

$$
\begin{aligned}
\left(\frac{1}{2}-\right. & \left.\frac{1}{n}\right) \frac{2 h_{n}}{g\left(x_{0}-h_{n}, x_{0}+h_{n}\right)} \frac{g\left(E \cap\left(x_{0}, x_{0}+h_{n}\right)\right)}{\left|E \cap\left(x_{0}, x_{0}+h_{n}\right)\right|}\left(\frac{w\left(x_{0}-h_{n}, x_{0}\right)}{h_{n}}\right)^{1 / q} \\
& \leq C h_{n}^{1 / p-1 / q}\left(\frac{v_{E}\left(x_{0}, x_{0}+h_{n}\right)}{h_{n}}\right)^{1 / p} .
\end{aligned}
$$

Inequality (2.5) shows that the sequence $\left\{E \cap\left(x_{0}, x_{0}+h_{n}\right)\right\}$ shrinks to $x_{0}$ nicely (see [8], p. 140). On taking $n \rightarrow \infty$, it follows from the Lebesgue differential theorem, more precisely its slightly generalized version (see [8], p. 141), that

$$
\frac{1}{2} w\left(x_{0}\right) \leq 0
$$

This contradicts the fact that $w\left(x_{0}\right)>\frac{1}{M}$.

Now we prove (ii). For contradiction we suppose

$$
|S|=\left|\left\{x \in(a, b): w(x)>(2 C)^{p} v(x)\right\}\right|>0 .
$$

Write $v_{s}(x)=v(x) \chi_{s}(x)$. Choose $x_{0} \in S$ in the same way as that in the proof of (i). Then the same argument as above shows

$$
\frac{1}{2} w\left(x_{0}\right)^{1 / p} \leq C v\left(x_{0}\right)^{1 / p}
$$

This contradicts the fact that $(2 C)^{p} v\left(x_{0}\right)<w\left(x_{0}\right)$.

Remark 6. It follows from Proposition 1 that we may assume that $w(x)$ is locally integrable on $R$ and $v(x)>0$ a.e. $x \in R$ in advance, when we study the weak type or strong type inequality for the one-sided maximal operator.

Proof of Theorem 3. In order to prove the first part, without loss of generality we may assume that $f \geq 0$ is bounded and has compact support. For $\lambda>0$, let $\Omega_{\lambda},\left(a_{j}, b_{j}\right)$ and $\left(x_{j}^{k}, x_{j}^{k+1}\right)$ be the same as those in the proof of the sufficiency of Theorem 1. Then we can write

$$
\begin{aligned}
\lambda^{q} w\left(\Omega_{\lambda}\right) & =\sum_{j} \sum_{k=0}^{\infty} \lambda^{q} w\left(x_{j}^{k}, x_{j}^{k+1}\right) \\
& =\sum_{j} \sum_{k=0}^{\infty} \lambda^{q} w\left(x_{j}^{2 k}, x_{j}^{2 k+1}\right)+\sum_{j} \sum_{k=0}^{\infty} \lambda^{q} w\left(x_{j}^{2 k+1}, x_{j}^{2 k+2}\right) \\
& =\mathrm{I}+\mathrm{II}, \quad \text { say. }
\end{aligned}
$$


Suppose $p>1$. We shall only estimate term I in (2.6), and the rest is similar.

$$
\begin{aligned}
\mathrm{I} & \leq 4^{q} \sum_{j} \sum_{k=0}^{\infty} \frac{w\left(x_{j}^{2 k}, x_{j}^{2 k+1}\right)}{g\left(x_{j}^{2 k}, x_{j}^{2 k+2}\right)^{q}}\left(\int_{x_{j}^{2 k+1}}^{x_{j}^{2 k+2}} f g\right)^{q} \quad(\text { by }(2.4)) \\
& \leq 4^{q} \sum_{j} \sum_{k=0}^{\infty} \frac{w\left(x_{j}^{2 k}, x_{j}^{2 k+1}\right)}{q\left(x_{j}^{2 k}, x_{j}^{2 k+2}\right)^{q}}\left(\int_{x_{j}^{2 k+1}}^{x_{j}^{2 k+2}} f^{p} v\right)^{q / p} \sigma\left(x_{j}^{2 k+1}, x_{j}^{2 k+2}\right)^{q / p^{\prime}}
\end{aligned}
$$

(by Hölder's inequality)

$$
\begin{aligned}
& \leq 4^{q}\left[\sum_{j} \sum_{k=0}^{\infty}\left(\frac{w\left(x_{j}^{2 k}, x_{j}^{2 k+1}\right)^{1 / q} \sigma\left(x_{j}^{2 k+1}, x_{j}^{2 k+2}\right)^{1 / p^{\prime}}}{g\left(x_{j}^{2 k}, x_{j}^{2 k+2}\right)}\right)^{r}\right]^{q / r} \\
& \times\left(\sum_{j} \sum_{k=0}^{\infty} \int_{x_{j}^{2 k+1}}^{x_{j}^{2 k+2}} f^{p} v\right)^{p / q} \quad \text { (by Hölder's inequality again) } \\
& \leq(4 B)^{q}\left(\sum_{j} \sum_{k=0}^{\infty} \int_{x_{j}^{2 k+1}}^{x_{j}^{2 k+2}} f^{p} v\right)^{q / p} .
\end{aligned}
$$

Thus we get

$$
\left(\lambda^{q} w\left(\Omega_{\lambda}\right)\right)^{1 / q} \leq 2^{1 / p^{\prime}} 4 B\left(\int_{R} r^{p} v\right)^{1 / p}
$$

With a few obvious changes (cf. the proof of the sufficiency of Theorem 1) the foregoing procedure is still available for the case of $p=1$. The first part of Theorem 3 is proved.

Now we prove the rest of Theorem 3. Let $p>1$. Given $\left\{\left(a_{j}, c_{j}\right)\right\}$ pairwise disjoint and $b_{j} \in\left(a_{j}, c_{j}\right)$, we may assume $0<\sigma\left(b_{j}, c_{j}\right)<\infty$ for all $j$. Indeed, if $\sigma\left(b_{j}, c_{j}\right)=\infty$ for some $j$, which means $\int_{R}\left(\frac{g}{v} \chi_{\left(b_{j}, c_{j}\right)}\right)^{p^{\prime}} v d x=\infty$, then there exists $f$ such that $\int_{R}|f(x)|^{p} v(x) d x$ and $\int_{b_{j}}^{c_{j}} f g=\infty$, which implies $M_{g}^{+} f(x)=\infty$ on $\left(-\infty, b_{j}\right)$. Therefore the strong type $(p, q)$ inequality holds only if $w(x)=0$ a.e. $x \in\left(-\infty, b_{j}\right)$, and we have $w\left(a_{j}, b_{j}\right)=0$. Let

$$
f_{j}(x)=\left(\frac{w\left(a_{j}, b_{j}\right)^{1 / q} \sigma\left(b_{j}, c_{j}\right)^{1 / q^{\prime}}}{g\left(a_{j}, c_{j}\right)}\right)^{r / p}\left(\frac{g(x)}{v(x)}\right)^{p^{\prime} / p} \chi_{\left(b_{j}, c_{j}\right)}(x)
$$

and $f_{N}(x)=\sum_{j=1}^{N} f_{j}(x)$ for given natural number $N$. Observe that

$$
M_{g}^{+} f(x) \geq \frac{1}{g\left(a_{j}, c_{j}\right)} \int_{b_{j}}^{c_{j}} f g \quad \text { for every } f \geq 0 \text { and } x \in\left(a_{j}, b_{j}\right)
$$


We have

$$
\begin{aligned}
C^{p} \sum_{j=1}^{N} & \left(\frac{w\left(a_{j}, b_{j}\right)^{1 / q} \sigma\left(b_{j}, c_{j}\right)^{1 / p^{\prime}}}{g\left(a_{j}, c_{j}\right)}\right)^{r}=C^{p} \int_{R} f_{N}(x)^{p} v(x) d x \\
& \geq\left(\int_{R}\left(M_{g}^{+} f_{N}(x)\right)^{q} w(x) d x\right)^{p / q} \\
& \geq\left(\sum_{j=1}^{N}\left[\frac{\int_{b_{j}}^{c_{j}} f_{N} g}{g\left(a_{j}, c_{j}\right)}\right]^{q} w\left(a_{j}, b_{j}\right)\right)^{p / q}(\text { by }(2.8)) . \\
& =\left\{\sum \frac{w\left(a_{j}, b_{j}\right)}{g\left(a_{j}, c_{j}\right)^{q}}\left[\left(\frac{w\left(a_{j}, b_{j}\right)^{1 / q} \sigma\left(b_{j}, c_{j}\right)^{1 / q^{\prime}}}{g\left(a_{j}, c_{j}\right)}\right)^{r / p} \sigma\left(b_{j}, c_{j}\right)\right]^{q}\right\}^{p / q} \\
& =\left\{\sum\left(\frac{w\left(a_{j}, b_{j}\right)^{1 / q} \sigma\left(b_{j}, c_{j}\right)^{1 / p^{\prime}}}{g\left(a_{j}, c_{j}\right)}\right)^{r}\right\}^{p / q} .
\end{aligned}
$$

This is

$$
\left[\sum_{j=1}^{N}\left(\frac{w\left(a_{j}, b_{j}\right)^{1 / q} \sigma\left(b_{j}, c_{j}\right)^{1 / p^{\prime}}}{g\left(a_{j}, c_{j}\right)}\right)^{r}\right]^{1 / r} \leq C .
$$

Letting $N \rightarrow \infty$, we get $(w, v) \in B_{g}^{+}(p, q)$ with $B \leq C$.

Suppose $p=1$. Keeping Remark 5 in mind, we assume

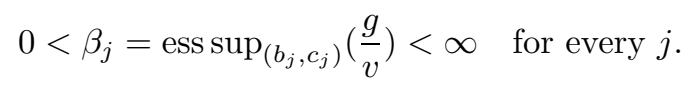

Given $\lambda>1$, set $E_{j}=\left\{x \in\left(b_{j}, c_{j}\right): \frac{g(x)}{v(x)}>\frac{\beta_{j}}{\lambda}\right\}$. Then $v\left(E_{j}\right)<\infty$ and $\left|E_{j}\right|>0$ which implies $v\left(E_{j}\right)>0$ (cf. Remark 6 we may assume $v(x)>0$ a.e.). Put

$$
f_{j}(x)=\left(\frac{w\left(a_{j}, b_{j}\right)^{1 / q}}{g\left(a_{j}, c_{j}\right)} \beta_{j}\right)^{r} \frac{\chi_{E_{j}}(x)}{v\left(E_{j}\right)}
$$

and $f_{N}(x)=\sum_{j=1}^{N} f_{j}(x)$. Then

$$
\int_{R} f_{N}(x) v(x) d x=\sum_{j=1}^{N}\left(\frac{w\left(a_{j}, b_{j}\right)^{1 / q}}{g\left(a_{j}, c_{j}\right)} \beta_{j}\right)^{r}
$$

and

$$
\begin{gathered}
{\left[\frac{\int_{b_{j}}^{c_{j}} f_{N} g}{g\left(a_{j}, c_{j}\right)}\right]^{q} w\left(a_{j}, b_{j}\right)=\frac{w\left(a_{j}, b_{j}\right)}{g\left(a_{j}, c_{j}\right)^{q}}\left[\left(\frac{w\left(a_{j}, b_{j}\right)^{1 / q}}{g\left(a_{j}, c_{j}\right)} \beta_{j}\right)^{r} \frac{g\left(E_{j}\right)}{v\left(E_{j}\right)}\right]^{q}} \\
\geq\left(\frac{1}{\lambda}\right)^{q}\left[\left(\frac{w\left(a_{j}, b_{j}\right)^{1 / q}}{g\left(a_{j}, c_{j}\right)} \beta_{j}\right)^{r} \frac{\beta_{j}}{g\left(a_{j}, c_{j}\right)}\right]^{q} w\left(a_{j}, b_{j}\right) \\
\quad\left(\text { since } g\left(E_{j}\right)>\frac{\beta_{j}}{\lambda} v\left(E_{j}\right)\right) \\
=\left(\frac{1}{\lambda}\right)^{q}\left(\frac{w\left(a_{j}, b_{j}\right)^{1 / q}}{g\left(a_{j}, c_{j}\right)} \beta_{j}\right)^{r} \quad\left(\text { since } \frac{1}{r}=\frac{1}{q}-1\right) .
\end{gathered}
$$


The argument similar to that used above shows

$$
\left[\sum_{j=1}^{N}\left(\frac{w\left(a_{j}, b_{j}\right)^{1 / q}}{g\left(a_{j}, c_{j}\right)} \beta_{j}\right)^{r}\right]^{1 / r} \leq \lambda C .
$$

Then we obtain $(w, v) \in B_{g}^{+}(1, q)$ with $B \leq C$. This completes the proof of Theorem 3.

Proof of Theorem 4. We shall prove the theorem only in the case $p>1$, since the necessary modifications for the case $p=1$ are indicated in the proof of Theorems 1 and 3.

(i) $\Rightarrow$ (ii) Similarly to the proof of Theorem 3, the condition $\sigma(a, b)=\infty$ together with the weak type inequality (1.5) implies that $w(x)=0$ for a.e. $x \in(-\infty, b)$. Therefore we may assume that $0<\sigma\left(b_{j}, c_{j}\right)<\infty$ for all $j$. Let

$$
f=\left\{\sum_{j}\left(\frac{g\left(a_{j}, c_{j}\right)}{\sigma\left(b_{j}, c_{j}\right)}\right)^{p} \frac{\sigma(x)}{v(x)} \chi_{\left(b_{j}, c_{j}\right)}\right\}^{1 / p} .
$$

It follows from (2.8) that $M_{g}^{+} f(x) \geq 1$ on $\bigcup\left(a_{j}, b_{j}\right)$ and the weak type inequality (1.5) yields

$$
w\left(\bigcup\left(a_{j}, b_{j}\right)\right)^{p / q} \leq C \int_{R} f^{p} v=C \sum_{j}\left(\frac{g\left(a_{j}, c_{j}\right)}{\sigma\left(b_{j}, c_{j}\right)^{1 / p^{\prime}}}\right)^{p} .
$$

This is (ii) with $B \leq C$.

(ii) $\Rightarrow$ (iii) For $\lambda>0$, write $E_{\lambda}=\{\Phi(x)>\lambda\}$. For each $x \in E_{\lambda}$ there exist $a_{x}<b_{x}<c_{x}$ such that $x \in\left(a_{x}, b_{x}\right)$ and $\frac{w\left(a_{x}, b_{x}\right)^{1 / p} \sigma\left(b_{x}, c_{x}\right)^{1 / p^{\prime}}}{g\left(a_{x}, c_{x}\right)}>\lambda$. Then for arbitrary compact set $K \subset E_{\lambda}$, from $\left\{\left(a_{x}, b_{x}\right)\right\}_{x \in K}$ we can choose a finite subfamily of open intervals $\left\{I_{i}\right\}$, which still covers $K$. By use of the covering lemma in [3] (Lemma 4.4, p. 25), one can select a finite subsequence $\left\{\left(a_{j}, b_{j}\right)\right\}$ from $\left\{I_{i}\right\}$ so that $\left\{\left(a_{j}, b_{j}\right)\right\}$ are pairwise disjoint and $w\left(\bigcup_{i} I_{i}\right) \leq 2 \sum_{j} w\left(a_{j}, b_{j}\right)$. For the set $\left\{\left(a_{j}, b_{j}\right)\right\}$, we have

$$
\begin{aligned}
w\left(\bigcup_{j}\left(a_{j}, b_{j}\right)\right)^{p / q} & \leq B^{p} \sum_{j}\left(\frac{g\left(a_{j}, c_{j}\right)}{\sigma\left(b_{j}, c_{j}\right)^{1 / p^{\prime}}}\right)^{p} \quad(\text { by }(\mathrm{ii})) \\
& \leq B^{p} \lambda^{-p} \sum_{j} w\left(a_{j}, b_{j}\right)
\end{aligned}
$$

Thus we get

$$
w(K) \leq w\left(\bigcup_{i} I_{i}\right) \leq 2 \sum_{j} w\left(a_{j}, b_{j}\right) \leq 2 B^{r} \lambda^{-r},
$$

which implies $\|\Phi\|_{r, \infty, w} \leq 2^{1 / r} B$.

(iii) $\Rightarrow$ (i) Let $f, \Omega_{\lambda}$ and $\left\{\left(x_{j}^{k}, x_{j}^{k+1}\right)\right\}$ be the same as those in the proof of Theorem 1. In addition, we may assume $\int_{R} f^{p} v=1$. Write

$$
\begin{aligned}
w\left(\Omega_{\lambda}\right) & =w\left(\Omega_{\lambda} \cap\left\{\Phi(x)>\lambda^{q / r}\right\}\right)+w\left(\Omega_{\lambda} \cap\left\{\Phi(x) \leq \lambda^{q / r}\right\}\right) \\
& =\mathrm{I}+\mathrm{II}, \quad \text { say. }
\end{aligned}
$$


For item I in (2.9) we have

$$
\mathrm{I} \leq w\left(\left\{\Phi(x)>\lambda^{q / r}\right\}\right) \leq\|\Phi\|_{r, \infty, w}^{r} \lambda^{-q} .
$$

To estimate II in (2.9), we consider $\left(x_{j}^{k}, x_{j}^{k+1}\right)$ having non-empty intersection with $\left\{\Phi(x) \leq \lambda^{q / r}\right\}$. It follows from (2.4) and Hölder's inequality that

$$
\begin{aligned}
w\left(x_{j}^{k}, x_{j}^{k+1}\right)^{1 / p} \lambda & \leq 4 \frac{w\left(x_{j}^{k}, x_{j}^{k+1}\right)^{1 / p} \sigma\left(x_{j}^{k+1}, x_{j}^{k+2}\right)^{1 / p^{\prime}}}{g\left(x_{j}^{k}, x_{j}^{k+2}\right)}\left(\int_{x_{j}^{k+1}}^{x_{j}^{k+2}} f^{p} v\right)^{1 / p} \\
& \leq 4 \lambda^{q / r}\left(\int_{x_{j}^{k+1}}^{x_{j}^{k+2}} f^{p} v\right)^{1 / p},
\end{aligned}
$$

since $\left(x_{j}^{k} x_{j}^{k+1}\right) \cap\left\{\Phi(x) \leq \lambda^{q / r}\right\} \neq \varnothing$. Raising (2.11) to the power of $p$ and then summing it over $j$ and $k$, we have

$$
\mathrm{II} \leq 4^{p} \lambda^{-q} .
$$

Combining (2.10) and (2.12), we obtain (1.5) with $C \leq\left(\|\Phi\|_{r, \infty, w}^{r}+4^{p}\right)^{1 / q}$. The proof of Theorem 4 is complete.

\section{ACKNOWLEDGMENT}

The author wishes to express many thanks to Dr. J. R. Partington for a lot of helpful conversations and suggestions. Thanks are also due to the referee for informing me of Verbitsky's paper [11] and suggesting to me to consider Verbitsky's type condition.

\section{REFERENCES}

1. R. R. Coifman and C. Fefferman, Weighted norm inequalities for maximal functions and singular integrals, Studia Math. 5 (1974), 241-250. MR 50:10670

2. J. Garcia-Cuerva and J. L. Rubio de Francia, Weighted norm inequalities and related topics, North-Holland, Amsterdam, 1985. MR 87d:42023

3. J. B. Garnett, Bounded analytic functions, Academic Press, New York, 1981. MR 83g:30037

4. Lai Qinsheng, Two weight mixed $\Phi$-inequalities for the one-sided maximal function, preprint.

5. F. J. Martin-Reyes, New proofs of weighted inequalities for the one-sided Hardy-Littlewood maximal functions, preprint. MR 93d:42016

6. F. J. Martin-Reyes, P. Ortega Salvador, and A. De La Torre, Weighted inequalities for onesided maximal functions, Trans. Amer. Math. Soc. 319 (1990), 517-534. MR 90j:42047

7. B. Muckenhoupt, Weighted norm inequalities for the Hardy maximal function, Trans. Amer. Math. Soc. 165 (1972), 207-227. MR 45:2461

8. W. Rudin, Real and complex analysis, 3rd ed., McGraw-Hill, New York, 1978.

9. E. Sawyer, Weighted inequalities for the one-sided Hardy-Littlewood maximal functions, Trans. Amer. Math. Soc. 297 (1986), 53-61. MR 87k:42018

10. W Weighted norm inequalities for fractional maximal operators, CMS Conf. Proc., vol. 1 Amer. Math. Soc., Providence, RI, 1981 pp. 283-309. MR 83k:42020a

11. I. E. Verbitsky, Weighted norm inequalities for maximal operators and Pisier's theorem on factorization through $L^{p, \infty}$, Integral Equations Operator Theory 15 (1992), 124-153. MR 93d: 42017

Department of Pure Mathematics, The University of Leeds, Leeds, LS2 9JT, England Current address: Department of Mathematics \& Statistics, University of Edinburgh, King's Buildings, Mayfield Road, Edinburgh EH9 3JZ, United Kingdom

E-mail address: pmt5lq@sun.leeds.ac.uk

E-mail address: qlai@maths.ed.ac.uk 